\title{
Constructing cubic curves with involutions
}

\section{Lorenz Halbeisen $^{1}$ (D) Norbert Hungerbühler ${ }^{1}$}

Received: 7 June 2021 / Accepted: 13 August 2021 / Published online: 3 September 2021

(c) The Author(s) 2021

\begin{abstract}
In 1888, Heinrich Schroeter provided a ruler construction for points on cubic curves based on line involutions. Using Chasles' Theorem and the terminology of elliptic curves, we give a simple proof of Schroeter's construction. In addition, we show how to construct tangents and additional points on the curve using another ruler construction which is also based on line involutions. As an application of Schroeter's construction we provide a new parametrisation of elliptic curves with torsion group $\mathbb{Z} / 2 \mathbb{Z} \times \mathbb{Z} / 8 \mathbb{Z}$ and give some configurations with all their points on a cubic curve.
\end{abstract}

Keywords Cubic curve $\cdot$ Line involution - Ruler constructions · Elliptic curve · Configurations

Mathematics Subject Classification 51A05 · 51A20

\section{Introduction}

Heinrich Schroeter gave in Schroeter (1888) a surprisingly simple ruler construction to generate points on a cubic curve. Since he did not provide a formal proof for the construction, we would like to present this here. Schroeter's construction can be interpreted as an iterated construction of line involutions. Thus, we first define the notion of a line involution with cross-ratios, and then we show how one can construct line involutions with ruler only.

For the sake of simplicity, we introduce the following terminology: For two distinct points $P$ and $Q$ in the plane, $P Q$ denotes the line through $P$ and $Q, \overline{P Q}$ denotes the distance between $P$ and $Q$, and for two distinct lines $l_{1}$ and $l_{2}, l_{1} \wedge l_{2}$ denotes the intersection point of $l_{1}$ and $l_{2}$. We tacitly assume that the plane is the real projective

Norbert Hungerbühler

norbert.hungerbuehler@math.ethz.ch

Lorenz Halbeisen

lorenz.halbeisen@math.ethz.ch

1 Department of Mathematics, ETH Zentrum, Rämistrasse 101, 8092 Zürich, Switzerland 
plane, and therefore, $l_{1} \wedge l_{2}$ is defined for any distinct lines $l_{1}$ and $l_{2}$. For the cross-ratio of four lines $a, b, x, y$ of a pencil we use the notation $\operatorname{cr}(a, b, x, y)$.

Line involution. Given a pencil. A line involution $\Lambda$ is a mapping which maps each line $l$ of the pencil to a so-called conjugate line $\bar{l}$ of the pencil, such that the following conditions are satisfied:

- $\Lambda$ is an involution, i.e., $\Lambda \circ \Lambda$ is the identity, in particular we have $\Lambda(\bar{l})=l$.

- Given three different pairs of conjugate lines $a, \bar{a}, b, \bar{b}, c, \bar{c}$, and let $l_{1}, l_{2}, l_{3}, l_{4}$ be four lines among $a, \bar{a}, b, \bar{b}, c, \bar{c}$ from three different pairs of conjugate lines, then

$$
\operatorname{cr}\left(l_{1}, l_{2}, l_{3}, l_{4}\right)=\operatorname{cr}\left(\bar{l}_{1}, \bar{l}_{2}, \bar{l}_{3}, \bar{l}_{4}\right) .
$$

Notice that any line involution is defined by two different pairs of conjugate lines. We shall use the following construction for line involutions (for the correctness of the construction see Chasles (Chasles 1989, Note X, §34, (28), p. 317)): Given two pairs $a, \bar{a}$ and $b, \bar{b}$ of conjugate lines which meet in $P$. Suppose, we want to find the conjugate line $\bar{d}$ of a line $d$ from the same pencil. Choose a point $D \neq P$ on $d$ and two lines through $D$ which meet $a$ and $b$ in the points $A$ and $B$, and $\bar{a}$ and $\bar{b}$ in the points $\bar{A}$ and $\bar{B}$, respectively (see Fig. 1). Let $\bar{D}=A \bar{B} \wedge \bar{A} B$. Then the conjugate line $\bar{d}$ of $d$ with respect to the line involution defined by $a, \bar{a}, b, \bar{b}$ is the line $P \bar{D}$.

Vice-versa, let $A, \bar{A}$ and $B, \bar{B}$ be two pairs of different points and $D=A B \wedge \bar{A} \bar{B}$, $\bar{D}=A \bar{B} \wedge \bar{A} B$. Then, for an arbitrary point $P \notin\{A, \bar{A}, B, \bar{B}, D, \bar{D}\}$, the lines $a=P A, \bar{a}=P \bar{A}, b=P B, \bar{b}=P \bar{B}$, and $d=P D, \bar{d}=P \bar{D}$ are conjugate lines.

Notice that this construction can be carried out using only a ruler.

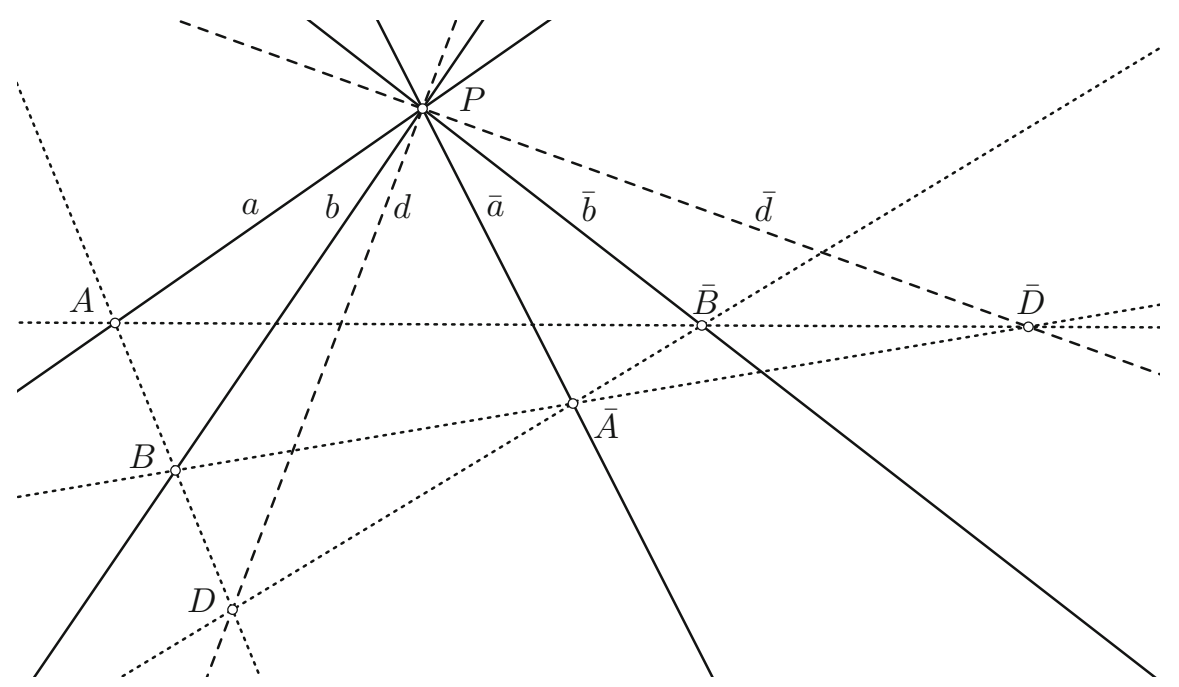

Fig. 1 Construction of conjugate lines 


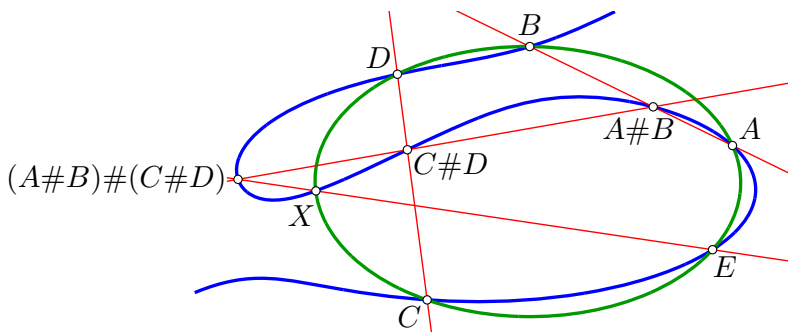

Fig. 2 Ruler construction of the point $X$

\section{Schroeter's construction for cubic curves}

Using the Braikenridge-Maclaurin Theorem, i.e., the converse of Pascal's theorem (see, for example, Coxeter and Greitzer 1967, p. 76), it is possible to construct an arbitrary number of points on a conic if five of its points are given, using only a ruler. An attempt to find a corresponding ruler construction for cubic curves was made in Mendelsohn et al. (1988): Let $A, B, C, D, E$ be five points on a cubic curve. Assume that the points $A \# B, C \# D$ and $(A \# B) \#(C \# D)$ are also known (see Fig. 2). Then, by Chasles' Theorem (see below), all cubic cuves through the eight points $A, B, C, D, E, A \# B, C \# D,(A \# B) \#(C \# D)$ pass through a ninth point $X$, namely the sixth intersection of the cubic curve with the conic through $A, B, C, D, E$. It is then shown that $X$ is also the intersection of the conic with the line through $E$ and $(A \# B) \#(C \# D)$, and can therefore be constructed with ruler alone. However the constructions in Mendelsohn et al. (1988) do not iterate and the authors were not aware of Schroeter's work, which allows to construct an arbitrary number of points on a cubic curve.

Schroeter's ruler construction, described in Schroeter (1888), is based on line involutions:

Schroeter's Construction. Let $A, \bar{A}, B, \bar{B}, C, \bar{C}$ be six pairwise distinct points in a plane such that no four points are collinear and the three pairs of points $A, \bar{A}, B, \bar{B}$, $C, \bar{C}$ are not the pairs of opposite vertices of the same complete quadrilateral. Now, for any two pairs of points $P, \bar{P}$ and $Q, \bar{Q}$, we define a new pair $S, \bar{S}$ of points by stipulating

$$
S:=P Q \wedge \bar{P} \bar{Q} \quad \text { and } \quad \bar{S}:=P \bar{Q} \wedge \bar{P} Q .
$$

Then all the points constructed in this way lie on a cubic curve.

Points $S, \bar{S}$ which are constructed by Schroeter's construction will be called Schroeter points or pairs of Schroeter points.

Notice first that with Schroeter's construction, we always construct pairs of conjugate lines: For any point $R \notin\{P, \bar{P}, Q, \bar{Q}, S, \bar{S}\}$ the lines $R P, R \bar{P}, R Q, R \bar{Q}, R S, R \bar{S}$ are pairs of conjugate lines with respect to the same line involution. Further notice that if the three pairs of points are opposite vertices of the same complete quadrilateral, then the construction gives us no additional points. 


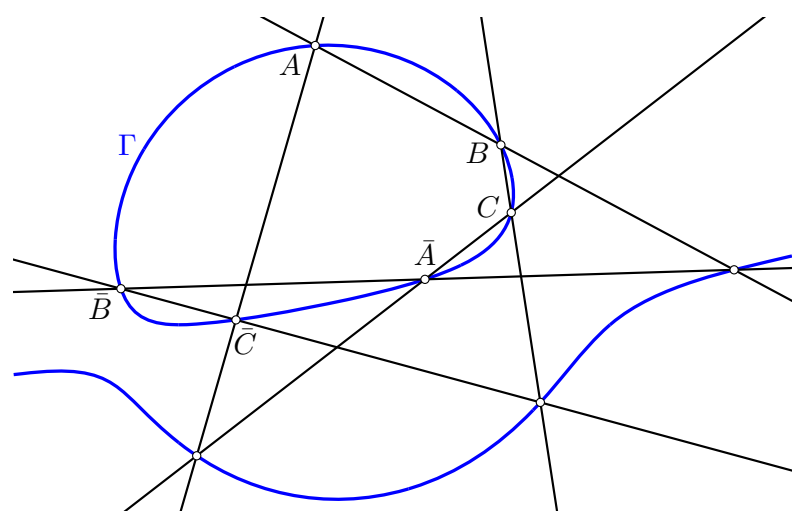

Fig. 3 Chasles' theorem in a Neuberg cubic $\Gamma$

At first glance, it is somewhat surprising that all the points we construct lie on the same cubic curve, which is defined by three pairs of points (recall that a cubic curve is defined by 9 points). The reason is that we have three pairs of points and not just 6 points. In fact, if we start with the same 6 points but pairing them differently, we obtain a different cubic curve. It is also not clear whether the construction generates infinitely many points of the curve. Schroeter claims in Schroeter (1888) that this is the case, but, as we will see in the next section, it may happen that the construction gives only a finite number of points.

\section{A proof of Schroeter's construction}

It is very likely that Schroeter discovered his construction based on his earlier work on cubics (see Schröter 1872, 1873). However, he did not give a rigorous proof of his construction, and the fact that he claimed wrongly that the construction generates always infinitely many points of the curve might indicate that he overlooked something. Below we give a simple proof of Schroeter's construction using Chasles' Theorem (see Chasles Chasles 1989, Chapitre IV, §8, p. 150) and the terminology of elliptic curves.

Theorem 1 (Chasles' Theorem) If a hexagon $A B C \bar{A} \bar{B} \bar{C}$ is inscribed in a cubic curve $\Gamma$ and the points $A B \wedge \bar{A} \bar{B}$ and $B C \wedge \bar{B} \bar{C}$ are on $\Gamma$, then also $C \bar{A} \wedge \bar{C} A$ is on $\Gamma$ (see Fig. 3).

With Chasles' Theorem we can prove the following

Proposition 2 Let $A, \bar{A}, B, \bar{B}, C, \bar{C}$ be six pairwise distinct points in a plane such that no four points are collinear and none of the pairs of points $A, \bar{A}, B, \bar{B}, C, \bar{C}$ is a pair of opposite vertices of the same complete quadrilateral. Furthermore, let

$$
\begin{array}{ll}
D:=A B \wedge \bar{A} \bar{B}, & E:=B C \wedge \bar{B} \bar{C}, \quad F:=C A \wedge \bar{C} \bar{A}, \\
\bar{D}:=A \bar{B} \wedge \bar{A} B, & \bar{E}:=B \bar{C} \wedge \bar{B} C, \quad \bar{F}:=C \bar{A} \wedge \bar{C} A,
\end{array}
$$


and assume that the 9 points $A, \bar{A}, B, \bar{B}, C, \bar{C}, D, E, F$ are pairwise distinct and that $\Gamma$ is a cubic curve passing through these 9 points. Then $\Gamma$ passes also through $\bar{D}, \bar{E}, \bar{F}$.

Proof Since the 6 points $A, \bar{A}, B, \bar{B}, C, \bar{C}$ as well as $D$ and $E$ are on $\Gamma$, by Chasles' Theorem we get that also $\bar{F}$ is on $\Gamma$. Similarly, since $C, \bar{C}, A, \bar{A}, B, \bar{B}, F, D$ are on $\Gamma$, also $\bar{E}$ is on $\Gamma$. Finally, since $B, \bar{B}, C, \bar{C}, A, \bar{A}, E, F$ are on $\Gamma$, also $\bar{D}$ is on $\Gamma$.

As an immediate consequence of Proposition 2 we get

Corollary 3 The unique cubic curve $\Gamma$ passing through the 9 points $A, \bar{A}, B, \bar{B}, C, \bar{C}$, $D, E, F$ contains also the 3 points $\bar{D}, \bar{E}, \bar{F}$.

In order to show that all the points constructed by Schroeter's construction lie on the same cubic curve, we interpret the construction in the setting of elliptic curves. For this, let $\Gamma$ be a cubic curve and let $\mathscr{O}$ be a point of inflection of $\Gamma$-recall that every cubic curve in the real projective plane has at least one point of inflection. For two points $P$ and $Q$ on $\Gamma$ let $P \# Q$ be the third intersection point (counting multiplicities) of $P Q$ with $\Gamma$, where for $P=Q, P Q$ is the tangent on $\Gamma$ with contact point $P$. Furthermore, for each point $P$ on $\Gamma$, let $-P:=\mathscr{O} \# P$. As usual, we define the binary operation + on the points of $\Gamma$ by stipulating

$$
P+Q:=-(P \# Q)
$$

Notice that $-P+P=\mathscr{O}$ and, since $\mathscr{O}$ is a point of inflection, we have $-\mathscr{O}=\mathscr{O}$. It is well known that the operation + is associative and the structure $(\Gamma, \mathscr{O},+)$ is an abelian group with neutral element $\mathscr{O}$, which is called an elliptic curve.

Now, let $\Gamma$ be the cubic curve passing through $A, \bar{A}, B, \bar{B}, C, \bar{C}, D, E, F$ and let $\mathscr{O}$ be a point of inflection of $\Gamma$. Then, by construction of $\Gamma$ we have, for example, $A \# B=\bar{A} \# \bar{B}$, or equivalently, $-(A+B)=-(\bar{A}+\bar{B})$.

Lemma 4 (a) Let $P, Q, \bar{P}, \bar{Q}$ be pairwise distinct points on a cubic curve $\Gamma$. If $S:=$ $P Q \wedge \bar{P} \bar{Q} \in \Gamma$ and $\bar{S}:=P \bar{Q} \wedge \bar{P} Q \in \Gamma$, then $P \# P=\bar{P} \# \bar{P} \in \Gamma, Q \# Q=$ $\bar{Q} \# \bar{Q} \in \Gamma$, and $S \# S=\bar{S} \# \bar{S} \in \Gamma$.

(b) Vice versa, if $P^{\prime}:=P \# P=\bar{P} \# \bar{P} \in \Gamma$ for two points $P, \bar{P} \in \Gamma$, then we have for all $Q \in \Gamma$ the following: If $S:=P \# Q$ and $\bar{Q}=S \# \bar{P}$, then $\bar{S}=P \bar{Q} \wedge \bar{P} Q \in \Gamma$ and $Q^{\prime}:=Q \# Q=\bar{Q} \# \bar{Q} \in \Gamma$.

Proof (a) By assumption we have $P \# Q=\bar{P} \# \bar{Q}=S$ and $P \# \bar{Q}=\bar{P} \# Q=\bar{S}$. With a point $\mathscr{O} \in \Gamma$ of inflection, we get

$$
P+Q=\mathscr{O} \#(P \# Q)=\mathscr{O} \#(\bar{P} \# \bar{Q})=\bar{P}+\bar{Q}
$$

and

$$
P+\bar{Q}=\mathscr{O} \#(P \# \bar{Q})=\mathscr{O} \#(\bar{P} \# Q)=\bar{P}+Q
$$




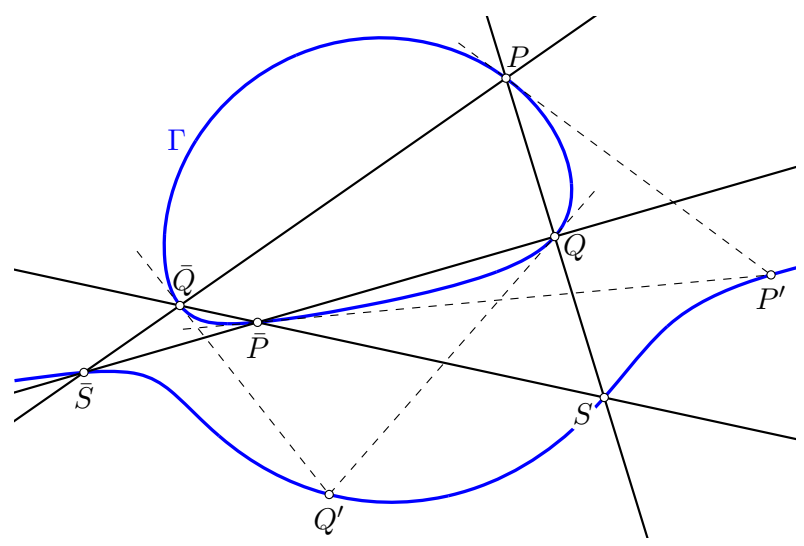

Fig. 4 Illustration Lemma 4

Adding (1) and (2) and subtracting $Q+\bar{Q}$ yields $P+P=\bar{P}+\bar{P}$ and hence $P \# P=\bar{P} \# \bar{P}$. Exchanging left and right hand in (1) and adding (2) gives, upon subtracting $P+\bar{P}, Q+Q=\bar{Q}+\bar{Q}$ and hence $Q \# Q=\bar{Q} \# \bar{Q} . S \# S=\bar{S} \# \bar{S}$ follows by exchanging the pair $Q, \bar{Q}$ by the pair $S, \bar{S}$.

(b) For the second part, we proceed as follows: By assumption, we have $P \# P=$ $\bar{P} \# \bar{P}$ and therefore $P+P=\mathscr{O} \#(P \# P)=\mathscr{O} \#(\bar{P} \# \bar{P})=\bar{P}+\bar{P}$. We add $S$ and subtract $P+\bar{P}$ to get $S+P-\bar{P}=S+\bar{P}-P$ or $(\mathscr{O} \#(S \# P)) \#(\mathscr{O} \# \bar{P})=(\mathscr{O} \#(S \# \bar{P})) \#(\mathscr{O} \# P)$. It follows that $(S \# P) \# \bar{P}=(S \# \bar{P}) \# P$, i.e., $Q \# \bar{P}=\bar{Q} \# P=\bar{S}$. Finally, $Q \# Q=\bar{Q} \# \bar{Q}=Q^{\prime}$ follows from the first part.

For the sake of simplicity we write $2 * P$ for $P+P$. Let $A, \bar{A}$ be a pair of points with $A \# A=\bar{A} \# \bar{A}$ on a cubic curve $\Gamma$, and with respect to a given point of inflection $\mathscr{O}$, let $T_{A}:=\bar{A}-A$. Then $A+T_{A}=\bar{A}$, which implies that

$$
2 * \bar{A}=2 *\left(A+T_{A}\right)=2 * A+2 * T_{A}
$$

Now, by assumption we have $2 * A=2 * \bar{A}$ and therefore we get that $2 * T_{A}=\mathscr{O}$. In other words, $T_{A}$ is a point of order 2 .

Now we are ready to prove the following

Theorem 5 All the points we obtain by Schroeter's construction belong to the same cubic curve.

Proof Let $A, \bar{A}, B, \bar{B}, C, \bar{C}$ be six pairwise distinct points in a plane such that no four points are collinear and none of the pairs of conjugate points $A, \bar{A}, B, \bar{B}, C, \bar{C}$ is a pair of opposite vertices of the same complete quadrilateral. Furthermore, let $D, \bar{D}, E, \bar{E}, F, \bar{F}$ be as in Proposition 2, and let $\Gamma$ be the cubic curve which passes through all of these 12 points. Finally, let $\mathscr{O}$ a fixed point of inflection of $\Gamma$, and let $T_{A}:=\bar{A}-A, T_{B}:=\bar{B}-B$, and $T_{C}:=\bar{C}-C$ be three points of order 2 . First we show that $T_{A}=T_{B}$. Since $A \# B=\bar{A} \# \bar{B}$ we have $-(A+B)=-\left(A+T_{A}+B+T_{B}\right)$, which implies that $T_{A}=T_{B}$. With a similar argument we obtain $T_{B}=T_{C}$. Thus, we have $T_{A}=T_{B}=T_{C}=: T$. 
We will say that a set $M$ of points is a good set, if

(a) all points of $M$ belong to $\Gamma$,

(b) the points $A, \bar{A}, B, \bar{B}, C, \bar{C}, D, \bar{D}, E, \bar{E}, F, \bar{F}$ belong to $M$,

(c) if the pair of points $S, \bar{S}$ belongs to $M$, then $S=P \# Q=\bar{P} \# \bar{Q}$ and $\bar{S}=P \# \bar{Q}=$ $\bar{P} \# Q$ for two pairs $P, \bar{P}$ and $Q, \bar{Q}$ in $M$,

(d) for all pairs $P, \bar{P}$ of $M$, we have $P \# P=\bar{P} \# \bar{P}$, and

(e) for all pairs $P, \bar{P}$ of $M$, we have $\bar{P}-P=T$.

Observe first, that $\{A, \bar{A}, B, \bar{B}, C, \bar{C}, D, \bar{D}, E, \bar{E}, F, \bar{F}\}$ is a good set. Indeed, (a) and (b) are trivially satisfied. The property (c) is clear for $D, \bar{D}, E, \bar{E}, F, \bar{F}$. For $A$ and $\bar{A}$ we have $A=B \# D=\bar{B} \# \bar{D}, \bar{A}=B \# \bar{D}=\bar{B} \# D$, and similarly for the pairs $B, \bar{B}$ and $C, \bar{C}$. The property (d) follows directly from Lemma 4(a). Finally, we have property (e) already for $A, \bar{A}, B, \bar{B}$ and $C, \bar{C}$. For $D$ the argument is similar: Let $T_{D}:=\bar{D}-D . T_{D}$ is a point of order 2 and from $B=A \# D=\bar{A} \# \bar{D}$ it follows $A+D=\bar{A}+\bar{D}=A+T+D+T_{D}$ and hence $T_{D}=T$. The analogous argument shows that $\bar{E}-E=\bar{F}-F=T$.

Now suppose that $M$ is a good set, and take two pairs $P, \bar{P}$ and $Q, \bar{Q}$ in $M$. Let $S=P Q \wedge \bar{P} \bar{Q}$ and $\bar{S}=P \bar{Q} \wedge \bar{P} Q$. Then we claim that $M \cup\{S, \bar{S}\}$ is also a good set. We first show that $P \# Q=\bar{P} \# \bar{Q}$ or equivalently that $P+Q=\bar{P}+\bar{Q}$. This is equivalent to $T=\bar{P}-P=Q-\bar{Q}=T$ which is true by property (e) for $M$ and the fact that $T$ is a point of order 2 . Then $P \# \bar{Q}=\bar{P} \# Q$ follows from Lemma 4(b). We conclude that the set $M \cup\{S, \bar{S}\}$ has the properties (a) and (c). Property (b) is trivial. For property (d) we need to see that $S \# S=\bar{S} \# \bar{S}$, which follows from Lemma 4(a). For property (e) we define $T_{S}=\bar{S}-S . T_{S}$ is a point of order 2 . From $Q=P \# S=\bar{P} \# \bar{S}$ it follows $P+S=\bar{P}+\bar{S}=P+T+S+T_{S}$ and hence $T_{S}=T$. This shows that $M \cup\{S, \bar{S}\}$ has all properties of a good set.

It follows that all points we obtain by Schroeter's construction belong to the same curve $\Gamma$.

The above proof shows that the Schroeter points have the following additional properties

- If $P, \bar{P}$ is a pair of Schroeter points on $\Gamma$, then the tangents in $P$ and $\bar{P}$ meet on $\Gamma$.

- With respect to a chosen point $\mathscr{O}$ of inflection, we have that $\bar{P}-P=T$ is a point of order 2 on $\Gamma$ which is the same for all Schroeter pairs $P, \bar{P}$.

The following result shows that we can construct the tangent to $\Gamma$ in each Schroeter point by a line involution (hence with ruler alone).

Proposition 6 Let $\Gamma$ be the cubic from Proposition 2. Assume that $S, \bar{S}, P, \bar{P}, Q, \bar{Q}$ are three of the pairs $A, \bar{A}, B, \bar{B}, C, \bar{C}, D, \bar{D}, E, \bar{E}, F, \bar{F}$ or of the pairs which are constructed by Schroeter's construction, such that $S P, S Q, S \bar{P}, S \bar{Q}$ are four distinct lines. Let $s=S \bar{S}$ and $\bar{S}$ its conjugate line with respect to the involution given by the lines $S P, S Q, S \bar{P}, S \bar{Q}$. Then $\bar{s}$ is tangent to $\Gamma$ in $S$ (see Fig. 5).

Before we can prove Proposition 6, we have to recall a few facts about cubic curves. It is well-known that every cubic curve can be transformed into Weierstrass Normal Form

$$
\Gamma_{a, b}: y^{2}=x^{3}+a x^{2}+b x
$$




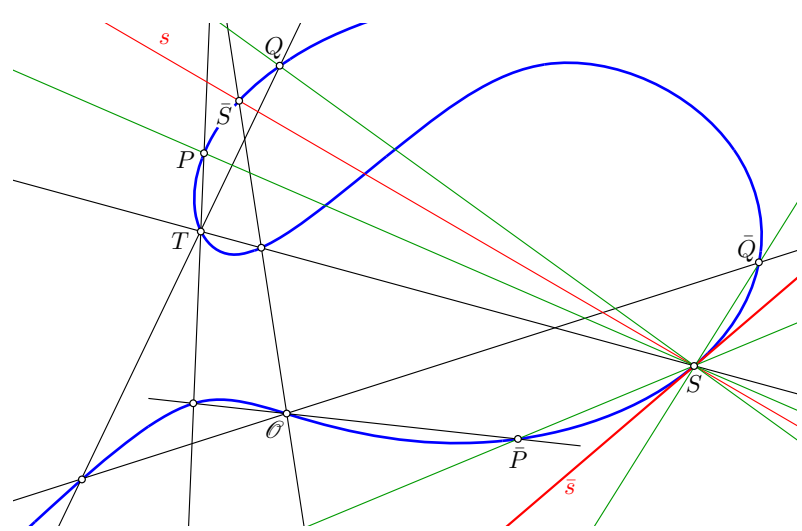

Fig. 5 Thin black lines: $\bar{P}=T+P, \bar{Q}=T+Q, \bar{S}=T+S$. The red lines $s=S \bar{S}$ and the thick tangent $\bar{s}$ in $S$ are conjugate lines with respect to the line involution given by the green lines $S P, S \bar{P}, S Q, S \bar{Q}$

with $a, b \in \mathbb{R}$. In the real projective plane, $\mathscr{O}=(0,1,0)$ is a point inflection of $\Gamma_{a, b}$ and $T_{a, b}=(0,0,1)$ is a point of order 2 of $\Gamma_{a, b}$, where $\mathscr{O}$ is the neutral element of the elliptic curve $\Gamma_{a, b}$. If $A$ is a point on $\Gamma_{a, b}$, then we call the point $\bar{A}:=T+A$ the conjugate of $A$. Since $T+T=\mathscr{O}$, we have

$$
\overline{\bar{A}}=T+\bar{A}=T+T+A=\mathscr{O}+A=A .
$$

Recall that $A \# B:=-(A+B)$. In particular, if $C=A \# A$, then the line through $C$ and $A$ is tangent to $\Gamma_{a, b}$ with contact point $A$.

The following result gives a connection between conjugate points and tangents.

Fact 7 If $A, \bar{A}, B$ are three points on $\Gamma_{a, b}$ which lie on a straight line, then $A \# A=\bar{B}$.

Proof If $A, \bar{A}, B$ are three points on $\Gamma_{a, b}$ on a straight line, then $A+\bar{A}=-B$. Thus, $A+T+A=T+A+A=-B$, which implies

$$
A+A=T+(T+A+A)=T+(-B)=(-T)+(-B)=-(T+B)=-\bar{B},
$$

and therefore, the line $A \bar{B}$ is tangent to $\Gamma_{a, b}$ with contact point $A$, i.e., $A \# A=\bar{B}$.

In homogeneous coordinates, the curve $y^{2}=x^{3}+a x^{2}+b x$ becomes

$$
\Gamma: Y^{2} Z=X^{3}+a X^{2} Z+b X Z^{2} .
$$

Assume now that $\tilde{A}=\left(r_{0}, r_{1}, 1\right)$ is a point on the cubic $\Gamma$, where $r_{0}, r_{1} \in \mathbb{R} \backslash\{0\}$. Then the point $(1,1,1)$ is on the curve

$$
r_{1}^{2} Y^{2} Z=r_{0}^{3} X^{3}+a r_{0}^{2} X^{2} Z+b r_{0} X Z^{2}
$$

Now, by exchanging $X$ and $Z$ (i.e., $(X, Y, Z) \mapsto(Z, Y, X))$, de-homogenising with respect to the third coordinate (i.e., $\left.(Z, Y, X) \mapsto\left(\frac{Z}{X}, \frac{Y}{X}, 1\right)\right)$, and multiplying 
with $\frac{1}{r_{1}^{2}}$, we obtain that the point $A=(1,1)$ is on the curve

$$
\Gamma_{\alpha, \beta, \gamma}: y^{2} x=\alpha+\beta x+\gamma x^{2} \text {, }
$$

where

$$
\alpha=\frac{r_{0}^{3}}{r_{1}^{2}}, \quad \beta=a \cdot \frac{r_{0}^{2}}{r_{1}^{2}}, \quad \gamma=b \cdot \frac{r_{0}}{r_{1}^{2}} .
$$

Notice that since $A=(1,1)$ is on $\Gamma_{\alpha, \beta, \gamma}$, we have $\alpha+\beta+\gamma=1$.

The next result gives a connection between line involutions and conjugate points.

Lemma 8 Let $A=\left(x_{0}, y_{0}\right)$ be an arbitrary but fixed point on $\Gamma_{\alpha, \beta, \gamma}$. For every point $P$ on $\Gamma_{\alpha, \beta, \gamma}$ which is different from $A$ and $\bar{A}$, let $g:=A P$ and $\bar{g}:=A \bar{P}$. Then the mapping $I_{A}: g \mapsto \bar{g}$ is a line involution.

Proof It is enough to show that there exists a point $\zeta_{0}$ (called the center of the involution) on the line $h: x=0$, such that the product of the distances between $\zeta_{0}$ and the intersections of $g$ and $\bar{g}$ with $h$ is constant.

Since $\bar{T}=T+T=\mathscr{O}$, with respect to $T$ we have $g: y=y_{0}$ and $\bar{g}: x=x_{0}$, which implies that $\zeta_{0}=\left(0, y_{0}\right)$. Now, let $P=\left(x_{1}, y_{1}\right)$ be a point on $\Gamma_{\alpha, \beta, \gamma}$ which is different from $A, \bar{A}, T, \mathscr{O}$, and let $g:=A P$ and $\bar{g}:=A \bar{P}$. Since $\bar{P}=\left(\frac{\alpha}{\gamma x_{1}},-y_{1}\right)$,

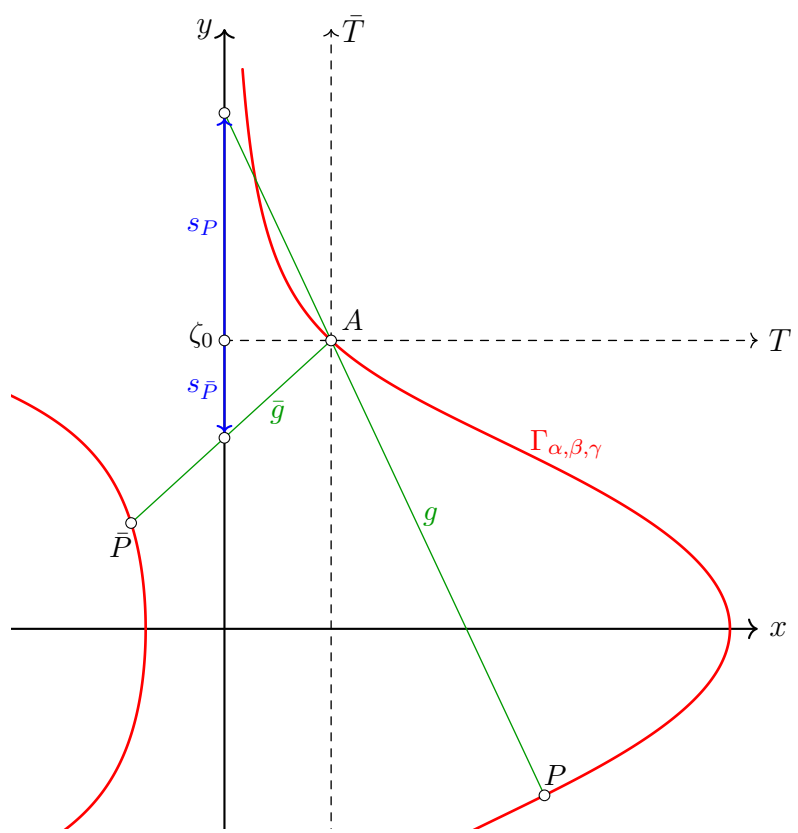

Fig. 6 Line involution 
the slopes $\lambda_{P}$ and $\lambda_{\bar{P}}$ of $g$ and $\bar{g}$, respectively, are

$$
\lambda_{P}=\frac{y_{1}-y_{0}}{x_{1}-x_{0}} \quad \text { and } \quad \lambda_{\bar{P}}=\frac{-y_{1}-y_{0}}{\frac{\alpha}{\gamma x_{1}}-x_{0}}
$$

Thus, the distances $s_{P}$ and $s_{\bar{P}}$ between $\zeta_{0}$ and the intersections of $g$ and $\bar{g}$ with $h$, respectively, are

$$
s_{P}=-\frac{x_{0}\left(y_{1}-y_{0}\right)}{x_{1}-x_{0}} \quad \text { and } \quad s_{\bar{P}}=\frac{x_{0}\left(y_{1}+y_{0}\right) \cdot \gamma x_{1}}{\alpha-\gamma x_{1} x_{0}} .
$$

Now,

$$
s_{P} \cdot s_{\bar{P}}=-\frac{x_{0}^{2}\left(y_{1}-y_{0}\right)\left(y_{1}+y_{0}\right) \gamma x_{1}}{\left(x_{1}-x_{0}\right)\left(\alpha-\gamma x_{0} x_{1}\right)}=-\frac{x_{0}^{2}\left(y_{1}^{2}-y_{0}^{2}\right) \gamma x_{1}}{\left(x_{1}-x_{0}\right)\left(\alpha-\gamma x_{0} x_{1}\right)},
$$

and using the fact that for $i \in\{0,1\}, y_{i}^{2}=\frac{\alpha}{x_{i}}+\beta+\gamma x_{i}$, we obtain

$$
s_{P} \cdot s_{\bar{P}}=\gamma \cdot x_{0}
$$

which is independent of the particular point $P=\left(x_{1}, y_{1}\right)$.

Since line involutions are invariant under projective transformations, as a consequence of Lemma 8 we obtain the following

Fact 9 Let $\Gamma$ be the cubic from Proposition 2 with two pairs of Schroeter points $P, \bar{P}=$ $T+P, Q, \bar{Q}=T+Q$, and let $R$ be a point on $\Gamma$ such that $R P, R \bar{P}, R Q, R \bar{Q}$ are four different lines. Let $S$ be a further point on $\Gamma$ and $\bar{S}=T+S$. Then the lines $s=R S$ and $\bar{s}=R \bar{S}$ are conjugate lines with respect to the line involution given by the lines $R P, R \bar{P}, R Q, R \bar{Q}$ (see Fig. 7).

Now we are ready to prove Proposition 6.

Proof of Proposition 6 First notice that $S$ and $\bar{S}$ are distinct, since otherwise, $\bar{S}=$ $T+S=S$, which implies that $T=S-S=\mathscr{O}$.

Assume that the line $s$ intersects $\Gamma$ in a point $U$ which is different from $S$ and $\bar{S}$. Then $\bar{U}:=T+U$ belongs to $\bar{s}$. If the line $\bar{s}$ intersects $\Gamma$ in a point $V$ which is different from $\bar{U}$, then, with respect to the involution given by the lines $\bar{U} S, \bar{U} P, \bar{U} \bar{S}, \bar{U} \bar{P}$, the point $\bar{V}$ belongs to $s$. Hence, $\bar{V}=\bar{S}$, which shows that $\bar{s}$ is tangent to $\Gamma$ in $S$.

Now, assume that the line $s$ intersects $\Gamma$ just in $S$ and $\bar{S}$. Then, the line $s$ is tangent to $\Gamma$ either in $S$ or in $\bar{S}$. We just consider the former case, the latter case is handled similarly. Let $P_{n}$ (for $n \in \mathbb{N}$ ) be a sequence of points on $\Gamma$ which are different from $S$ and which converges to $S$, i.e., $\lim _{n \rightarrow \infty} P_{n}=S$. Since for each $n \in \mathbb{N}$ we have $\bar{P}_{n}=T+P_{n}$ (where $\bar{P}_{n}:=T+P$ ), by continuity of addition we have $\lim _{n \rightarrow \infty} \bar{P}_{n}=\bar{S}$. For each $n \in N$ let $t_{n}:=P_{n} S$. Then, for each $n \in \mathbb{N}, \bar{t}_{n}=\bar{P}_{n} S$. Since $s$ is tangent to $\Gamma$ in $S$, by continuity, on the one hand we have $\lim _{n \rightarrow \infty} t_{n}=s$, and on the other hand we have $\lim _{n \rightarrow \infty} \bar{t}_{n}=s$, which implies that $\bar{s}=s$ and shows that $\bar{s}$ is tangent to $\Gamma$ in $S$. 


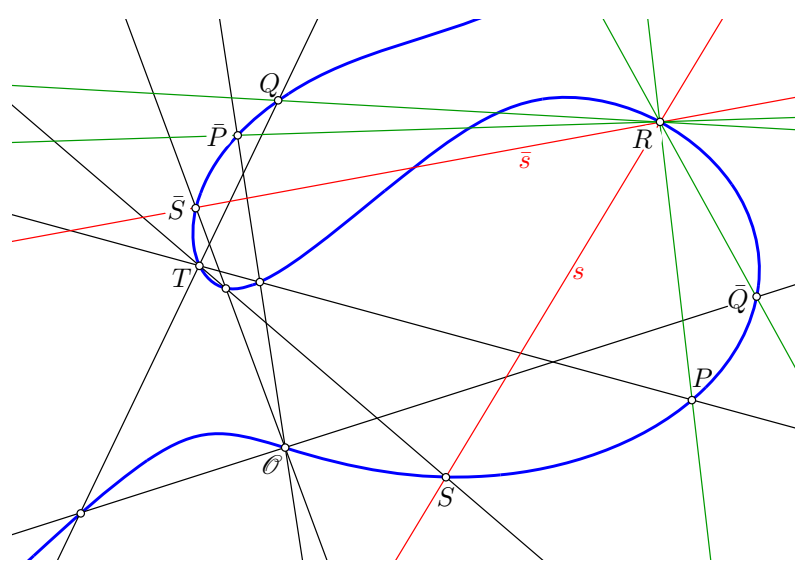

Fig. 7 Thin black lines: $\bar{P}=T+P, \bar{Q}=T+Q, \bar{S}=T+S$. The red lines $s=R S$ and $\bar{s}=R \bar{S}$ are conjugate lines with respect to the line involution given by the green lines $R P, R \bar{P}, R Q, R \bar{Q}$

As a corollary of Proposition 6 and Lemma 4(a) we obtain the following:

Corollary 10 Let $\Gamma$ be the cubic from Proposition 2. Then we have:

(a) In each Schroeter point it is possible to construct the tangent by a line involution, i.e., with a ruler construction.

(b) In addition to the Schroeter points on $\Gamma$ one can construct for each Schroeter pair $P, \bar{P}$ the point $P \# P=\bar{P} \# \bar{P} \in \Gamma$ by ruler alone: These are the intersection points of the tangents in $P$ and in $\bar{P}$.

Figure 8 shows a sample of Schroeter points and of intersection points of the corresponding tangents.

A priori it might be possible that Schroeter's construction does not yield all cubic curves. However, the next theorem says that in fact all cubic curves carry Schroeter's construction.

Theorem 11 Let $\Gamma$ be a non-singular cubic curve. Let $A, B, C$ be three different arbitrary points on $\Gamma$. Then, there are points $\bar{A}, \bar{B}, \bar{C}$ on $\Gamma$ such that $D=A B \wedge \bar{A} \bar{B}$, $E=B C \wedge \bar{B} \bar{C}, F=C A \wedge \bar{C} \bar{A}$ are points on $\Gamma$ and so do all the points given by Schroeter's construction.

Proof Choose $\bar{A}$ such that $A \# A=\bar{A} \# \bar{A}$ and $\bar{B}:=\bar{A} \#(A \# B)$. In particular, we have $A \# B=\bar{A} \# \bar{B}$, and, by Lemma $4, A \# \bar{B}=\bar{A} \# B$ and $B \# B=\bar{B} \# \bar{B}$. Let $\bar{C}:=\bar{B} \#(B \# C)$. In particular, we have $B \# C=\bar{B} \# \bar{C}$, and, by Lemma 4 , $B \# \bar{C}=\bar{B} \# C$ and $C \# C=\bar{C} \# \bar{C}$. It follows from Chasles' Theorem 1 that $A \# \bar{C}=\bar{A} \# C$. From the above, we obtain by applying Proposition 2 with $C$ and $\bar{C}$ exchanged, that $A \# C=\bar{A} \# \bar{C}$. Hence all points constructed from these points by Schroeter's construction lie on $\Gamma$.

Remarks. Let $\Gamma_{0}$ be the cubic curve passing through $A, \bar{A}, B, \bar{B}, C, \bar{C}, D, E, F$, let $\mathscr{O}$ be a point of inflection of $\Gamma_{0}$, and let $E_{0}=\left(\Gamma_{0}, \mathscr{O},+\right)$ be the corresponding elliptic curve. 


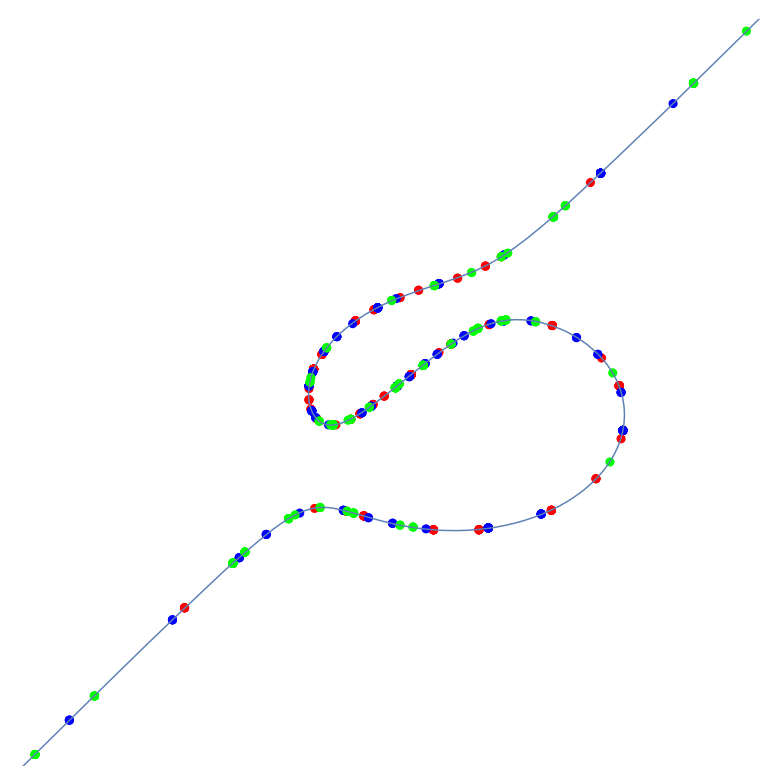

Fig. 8 Schroeter pairs $P$ (red), $\bar{P}$ (blue), and intersection points $P \# P=\bar{P} \# \bar{P}$ of the corresponding tangents (green)

(1) If $C_{n}$ is a cyclic group of order $n$, then there is a point on $\Gamma_{0}$ of order $n$ (with respect to $E_{0}$ ). This implies that if we choose the six starting points in a finite subgroup of $E_{0}$, then Schroeter's construction "closes" after finitely many steps and we end up with just finitely many points. However, if our 6 starting points are all rational and we obtain more than 16 points with Schroeter's construction, then, by Mazur's Theorem, we obtain infinitely many rational points on the cubic curve $\Gamma_{0}$.

(2) If the elliptic curve $E_{0}$ has three points of order 2 , then one of them, say $T$, has the property that for any point $P$ on $\Gamma_{0}$ we have $\bar{P}=P+T$. In particular, we have $\bar{T}=T+T=\mathscr{O}$. Furthermore, for the other two points of order 2, say $S_{1}$ and $S_{2}$, we have $S_{1}=S_{2}+T$ and $S_{2}=S_{1}+T$, i.e., $S_{1}=\bar{S}_{2}$.

(3) If we choose another point of inflection $\mathscr{O}^{\prime}$ on the cubic curve $\Gamma_{0}$, we obtain a different elliptic curve $E_{0}^{\prime}$. In particular, we obtain different inverses of the constructed points, even though the constructed points are exactly the same (see Fig. 9).

Example. Let $A, \bar{A}, B, \bar{B}, C, \bar{C}$ be six different starting points for Schroeter's construction such that no three points are co-linear. By a projective transformation, we can move $A \mapsto(0,0,1), \bar{A} \mapsto(0,1,0), B \mapsto(1,0,0), \bar{B} \mapsto(1,1,1), C \mapsto\left(C_{x}, C_{y}, 1\right)$, $\bar{C} \mapsto\left(\bar{C}_{x}, \bar{C}_{y}, 1\right)$. Then, the corresponding cubic curve $\Gamma$ we obtain by Schroeter's construction is given by the following equation:

$$
\begin{aligned}
& \Gamma: \quad x y^{2}-x^{2} y+x^{2} C_{y} \bar{C}_{y}+y^{2}\left(C_{x} \bar{C}_{x}-C_{x}-\bar{C}_{x}\right) \\
& \quad+x y\left(C_{x}+\bar{C}_{x}-C_{y} \bar{C}_{x}-C_{x} \bar{C}_{y}\right)-x C_{y} \bar{C}_{y}+y\left(C_{y} \bar{C}_{x}+C_{x} \bar{C}_{y}-C_{x} \bar{C}_{x}\right)=0
\end{aligned}
$$




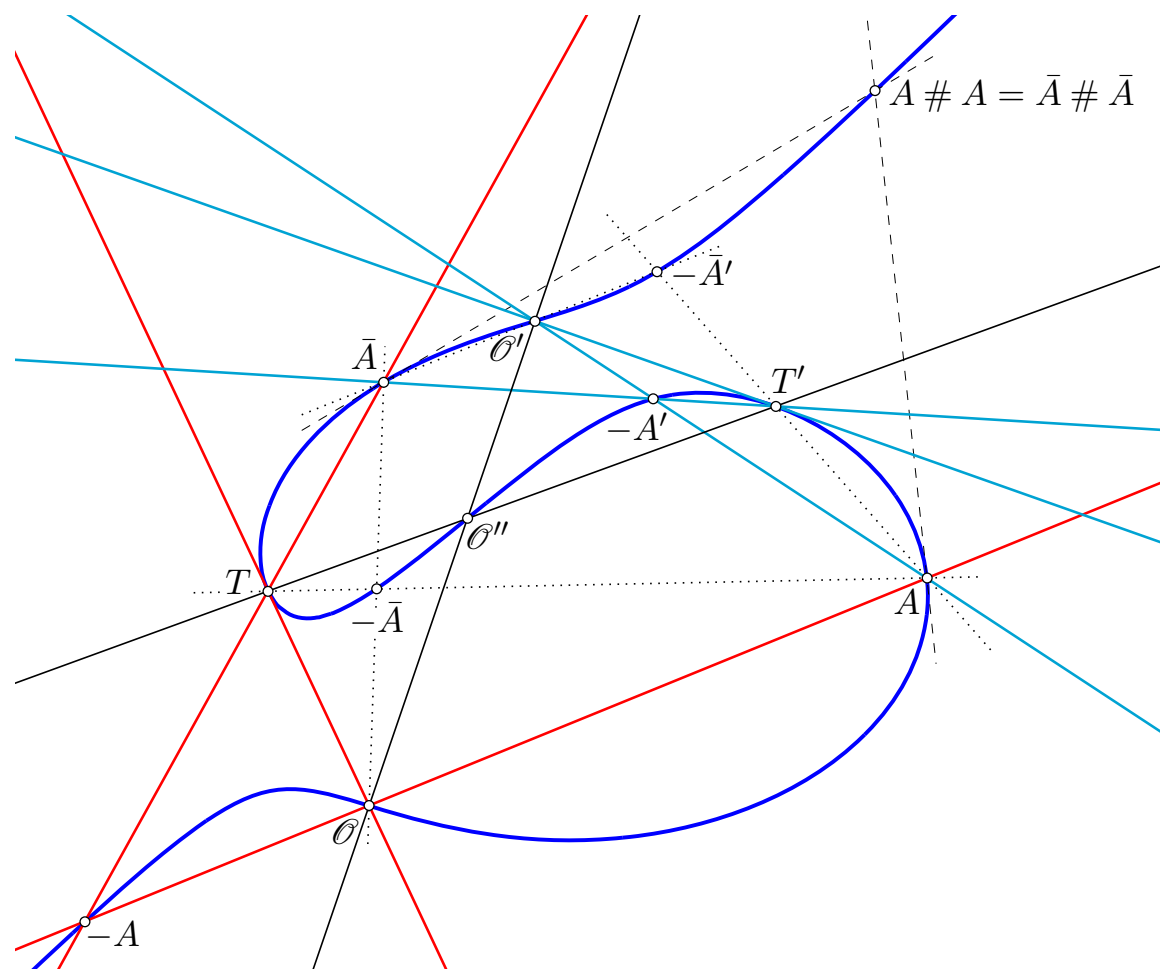

Fig. $9 A, \bar{A}$ is a Schroeter pair, in particular $A \# A=\bar{A} \# \bar{A}$. With respect to $\mathscr{O}$, we get the points $-A,-\bar{A}$, and the point $T=\bar{A}-A=A-\bar{A}$ of order 2 . With respect to $\mathscr{O}^{\prime}$, we get the points $-A^{\prime},-\bar{A}^{\prime}$, and the point $T^{\prime}=\bar{A}-A^{\prime}=A-\bar{A}^{\prime}$ of order 2 . The three points $\mathscr{O}, \mathscr{O}^{\prime}, \mathscr{O}^{\prime \prime}$ of inflection are collinear. The lines $T T^{\prime}$ and $\mathscr{O} \mathscr{O}^{\prime}$ meet in $\mathscr{O}^{\prime \prime}$

\section{Elliptic curves with Torsion group $\mathbb{Z} / 2 \mathbb{Z} \times \mathbb{Z} / 8 \mathbb{Z}$}

As a first application of Schroeter's construction we provide a new parametrisation of elliptic curves with torsion group $\mathbb{Z} / 2 \mathbb{Z} \times \mathbb{Z} / 8 \mathbb{Z}$. This parametrisation was the nucleus of the characterisation of elliptic curves with torsion group $\mathbb{Z} / 2 \mathbb{Z} \times \mathbb{Z} / 8 \mathbb{Z}$ and positive rank given in Halbeisen and Hungerbühler (2021). For other new parametrisationswhich are different to the parametrisations given by Kubert Kubert (1976) and Rabarison Rabarison (2010) — of elliptic curves with torsion group $\mathbb{Z} / 10 \mathbb{Z}, \mathbb{Z} / 12 \mathbb{Z}$, and $\mathbb{Z} / 14 \mathbb{Z}$ obtained by Schroeter's construction see Halbeisen et al. (2021).

Let $\Gamma_{a, b}: y^{2}=x^{3}+a x^{2}+b x$ be a regular curve with torsion group $\mathbb{Z} / 2 \mathbb{Z} \times \mathbb{Z} / 8 \mathbb{Z}$ over $\mathbb{Q}$. In homogeneous coordinates, the curve $\Gamma_{a, b}$ becomes

$$
\Gamma: Y^{2} Z=X^{3}+a X^{2} Z+b X Z^{2} .
$$

Assume now that $\tilde{A}=\left(\frac{n_{0}}{m_{0}}, \frac{n_{1}}{m_{1}}, 1\right)$ is a rational point on the cubic $\Gamma$, where $n_{0}, m_{0}, n_{1}, m_{1}$ are integers and $n_{0} \neq 0 \neq n_{1}$. Then the point $(1,1,1)$ is on the 
curve

$$
\frac{n_{1}^{2}}{m_{1}^{2}} Y^{2} Z=\frac{n_{0}^{3}}{m_{0}^{3}} X^{3}+a \frac{n_{0}^{2}}{m_{0}^{2}} X^{2} Z+b \frac{n_{0}}{m_{0}} X Z^{2}
$$

Now, by exchanging $X$ and $Z$ (i.e., $(X, Y, Z) \mapsto(Z, Y, X))$, de-homogenising with respect to the third coordinate (i.e., $\left.(Z, Y, X) \mapsto\left(\frac{Z}{X}, \frac{Y}{X}, 1\right)\right)$, and multiplying with $\frac{m_{1}^{2}}{n_{1}^{2}}$, we obtain that the point $A=(1,1)$ is on the curve

$$
\Gamma_{\alpha, \beta, \gamma}: y^{2} x=\alpha+\beta x+\gamma x^{2}
$$

where

$$
\alpha=\frac{n_{0}^{3} \cdot m_{1}^{2}}{m_{0}^{3} \cdot n_{1}^{2}}, \quad \beta=a \cdot \frac{n_{0}^{2} \cdot m_{1}^{2}}{m_{0}^{2} \cdot n_{1}^{2}}, \quad \gamma=b \cdot \frac{n_{0} \cdot m_{1}^{2}}{m_{0} \cdot n_{1}^{2}}
$$

Notice that since $A=(1,1)$ is on $\Gamma_{\alpha, \beta, \gamma}$, we have $\alpha+\beta+\gamma=1$, and recall that if $\tilde{A}=\tilde{B}+\tilde{B}$ for some rational point $\tilde{B}$ on $\Gamma$, then $n_{0}$ and $m_{0}$ are perfect squares.

In homogeneous coordinates, the neutral element of $\Gamma_{\alpha, \beta, \gamma}$ is $\mathscr{O}=(0,1,0)$ and the image under $\Phi$ of the point $(0,0,1)$ on $\Gamma_{a, b}$ is $T=(1,0,0)$. With respect to the curve $\Gamma_{\alpha, \beta, \gamma}$, we can compute the conjugate of a point by the following

Fact 12 Let $P=\left(x_{1}, y_{1}\right)$ be a point on $\Gamma_{\alpha, \beta, \gamma}$. Then

$$
\bar{P}=\left(\frac{\alpha}{\gamma x_{1}},-y_{1}\right) .
$$

Proof Let $P=\left(x_{1}, y_{1}\right)$ be a point on $\Gamma_{\alpha, \beta, \gamma}$. Then

$$
y_{1}^{2}=\frac{\alpha}{x_{1}}+\beta+\gamma x_{1}
$$

which implies that $x_{1}$ is a root of

$$
x^{2} \gamma+x\left(\beta-y_{1}^{2}\right)+\alpha=\frac{\left(x-x_{1}\right)\left(x \cdot \gamma x_{1}-\alpha\right)}{x_{1}} .
$$

The other root is $\frac{\alpha}{\gamma x_{1}}$, and hence, $\left(\frac{\alpha}{\gamma x_{1}}, y_{1}\right)$ is a point on $\Gamma_{\alpha, \beta, \gamma}$. Now, since $\bar{P}=T+P$ and $T=(1,0,0)$, we have $\bar{P}=\left(x_{2},-y_{1}\right)$, and therefore, $\bar{P}=\left(\frac{\alpha}{\gamma x_{1}},-y_{1}\right)$.

Let $\tilde{A}$ be a rational point on $\Gamma_{a, b}$ of order 4 , and let $\tilde{B}$ be such that $\tilde{B}+\tilde{B}=\tilde{A}$. Furthermore, let $\tilde{C}=\tilde{A}+\tilde{B}$, let $\tilde{T}=(0,0)$, and let $\tilde{S}$ be another rational point of order 2 . Finally, for a point $\tilde{P}$ on $\Gamma_{a, b}$, define $\tilde{P}_{1}:=\tilde{S}+\tilde{P}$. Now, there is a rational projective transformation $\Phi$ which maps the point $\tilde{A}$ to the point $A=(1,1)$ and the curve $\Gamma_{a, b}$ to the curve $\Gamma_{\alpha, \beta, \gamma}$. Notice that since $\tilde{B}+\tilde{B}=\tilde{A}, \alpha$ is a square, say $\alpha=\left(\frac{p}{q}\right)^{2}$ for some $\frac{p}{q} \in \mathbb{Q}$. 
Let $T:=\Phi(\tilde{T}), B:=\Phi(\tilde{B}), C:=\Phi(\tilde{C})$, and $S:=\Phi(\tilde{S})$. Then, for $A,-A, \bar{A}, \ldots$ we obtain the following correspondence between these points on $\Gamma_{\alpha, \beta, \gamma}$ and the elements of the group $\mathbb{Z} / 2 \mathbb{Z} \times \mathbb{Z} / 8 \mathbb{Z}$ :

\begin{tabular}{lllllllll}
\hline $\mathbb{Z} / 2 \mathbb{Z} \times \mathbb{Z} / 8 \mathbb{Z}$ & $(0,0)$ & $(0,1)$ & $(0,2)$ & $(0,3)$ & $(0,4)$ & $(0,5)$ & $(0,6)$ & $(0,7)$ \\
$\Gamma_{\alpha, \beta, \gamma}$ & $\mathscr{O}$ & $B$ & $A$ & $C$ & $T$ & $\bar{B}$ & $\bar{A}$ & $\bar{C}$ \\
$\Gamma_{\alpha, \beta, \gamma}$ & $S$ & $B_{1}$ & $A_{1}$ & $C_{1}$ & $\bar{S}$ & $\bar{B}_{1}$ & $\bar{A}_{1}$ & $\bar{C}_{1}$ \\
$\mathbb{Z} / 2 \mathbb{Z} \times \mathbb{Z} / 8 \mathbb{Z}$ & $(1,0)$ & $(1,1)$ & $(1,2)$ & $(1,3)$ & $(1,4)$ & $(1,5)$ & $(1,6)$ & $(1,7)$ \\
\hline
\end{tabular}

Let us now compute $\gamma$.

Lemma $13 \gamma=\alpha$.

Proof First, notice that $\bar{A}=-A$, and since $A=(1,1)$ we have $\bar{A}=\left(\frac{\alpha}{\gamma},-1\right)$. Thus, $\frac{\alpha}{\gamma}=1$ which implies $\alpha=\gamma$.

By considering the points $S$ and $\bar{S}$, we obtain the following result.

Lemma 14 If $\alpha=\left(\frac{p}{q}\right)^{2}$ for $\frac{p}{q} \in \mathbb{Q}$, then there are $r, s \in \mathbb{N}$ with $(r, s)=1$ such that

$$
p= \pm r s \text { and } q= \pm\left(r^{2}+s^{2}\right) .
$$

Proof Let $\alpha=\gamma=u^{2}$ and $u=\frac{p}{q}$. Since the $y$-coordinate of the points $S$ and $\bar{S}$ equals 0 , for $S=(x, 0)$ we have $u^{2}+\left(1-2 u^{2}\right) x+u^{2} x^{2}=0$. Hence,

$$
x=\frac{2 u^{2}-1 \pm \sqrt{1-4 u^{2}}}{2 u^{2}}
$$

and since $x \in \mathbb{Q}$, we have $1-4 u^{2}=\square$. Thus,

$$
1-\frac{4 p^{2}}{q^{2}}=\frac{q^{2}-4 p^{2}}{q^{2}}=\square,
$$

which implies $q^{2}-4 p^{2}=q^{2}-(2 p)^{2}=\square$. Since $(p, q)=1$, there are some $r, s \in \mathbb{N}$ with $(r, s)=1$ such that $p= \pm r s$ and $q= \pm\left(r^{2}+s^{2}\right)$.

Using the fact that $B \# B=-A$, we can show the following result.

Lemma 15 For $p= \pm r s$ and $q= \pm\left(r^{2}+s^{2}\right)$ we find $m, n \in \mathbb{N}$ such that $r=2 m n$ and $s=m^{2}-n^{2}$.

Proof First notice that $B \# B=-A$ and that $-\bar{A}=A=(1,1)$. Let $B=\left(x_{2}, y_{2}\right)$. Then, since $\alpha=\gamma, \bar{B}=\left(\frac{1}{x_{2}},-y_{2}\right)$. Since $B \# B=-A$, the points $B, \bar{B}, A$ are collinear, which implies that

$$
y_{2}=-\frac{x_{2}+1}{x_{2}-1} .
$$


Now, since $B$ is on the curve, we have

$$
y_{2}^{2} x_{2}=\alpha+(1-2 \alpha) x_{2}+\alpha x_{2}^{2},
$$

and for $\alpha=\frac{r^{2} s^{2}}{\left(r^{2}+s^{2}\right)}$ we obtain

$$
x_{2}=\frac{ \pm r^{2}+r s \pm s^{2} \pm(r-s) \sqrt{r^{2}+s^{2}}}{r s} .
$$

So, since $x_{2} \in \mathbb{Q}$, we have $r^{2}+s^{2}=\square$, which implies that there are $m, n \in \mathbb{N}$ with $(m, n)=1$ such that $r=2 m n$ and $s=m^{2}-n^{2}$.

Now, we are ready to give a parametrisation of elliptic curves with torsion group $\mathbb{Z} / 2 \mathbb{Z} \times \mathbb{Z} / 8 \mathbb{Z}$.

Theorem 16 Let $\frac{m}{n} \neq 1$ be a positive rational in lowest terms and let

$$
a_{1}=(2 m n)^{4}+\left(m^{2}-n^{2}\right)^{4}, b_{1}=(2 m n)^{4} \cdot\left(m^{2}-n^{2}\right)^{4} .
$$

Then the curve

$$
\Gamma_{a_{1}, b_{1}}: y^{2}=x^{3}+a_{1} x^{2}+b_{1} x
$$

is an elliptic curve with torsion group $\mathbb{Z} / 2 \mathbb{Z} \times \mathbb{Z} / 8 \mathbb{Z}$. Conversely, if $\Gamma_{a, b}$ is a regular elliptic curve with torsion group $\mathbb{Z} / 2 \mathbb{Z} \times \mathbb{Z} / 8 \mathbb{Z}$, then there exists a positive rational $\frac{m}{n}$ such that $\Gamma_{a, b}$ is isomorphic to $\Gamma_{a_{1}, b_{1}}$.

Proof By construction, for any relatively prime positive integers $m$ and $n$, the corresponding elliptic curve $\Gamma_{a_{1}, b_{1}}$ has torsion group $\mathbb{Z} / 2 \mathbb{Z} \times \mathbb{Z} / 8 \mathbb{Z}$.

On the other hand, if $\Gamma_{a, b}$ is an elliptic curve with torsion group $\mathbb{Z} / 2 \mathbb{Z} \times \mathbb{Z} / 8 \mathbb{Z}$, then we find a rational point $\tilde{A}$ on $\Gamma_{a, b}$ of order 4 , and by construction we find relatively prime positive integers $m$ and $n$ such that $\Gamma_{a, b}$ is isomorphic to the curve $\Gamma_{a_{1}, b_{1}}$.

As a last remark we would like to mention that for any positive integers $m$ and $n$ we have $\left(m^{2}-n^{2}\right)^{2}+(2 m n)^{2}=\square$, i.e., for $k=m^{2}-n^{2}$ and $l=2 m n,(k, l)$ is a so-called pythagorean pair. Now, in Halbeisen and Hungerbühler (2021) it is shown that the corresponding elliptic curve $\Gamma_{a_{1}, b_{1}}$ has positive rank over $\mathbb{Q}$ if and only if there exists a pythagorean pair $(r, s)$ such that $\left(k^{2} \cdot r, l^{2} \cdot s\right)$ is a pythagorean pair.

\section{Configurations on elliptic curves}

In the complex projective plane, a generic elliptic curve has nine inflection points, located in groups of three on a total of 12 lines. The nine inflection points and 12 lines form the so-called Hesse configuration $\left(9_{4}, 12_{3}\right)$ : See Fig. 10.

Recall that a $\left(p_{\lambda}, \ell_{\pi}\right)$ configuration consists of $p$ points and $\ell$ lines in the real (or complex) projective plane arranged in such a way that each of the $p$ points is 
Fig. 10 The Hesse configuration $\left(9_{4}, 12_{3}\right)$ : A model of a finite affine plane

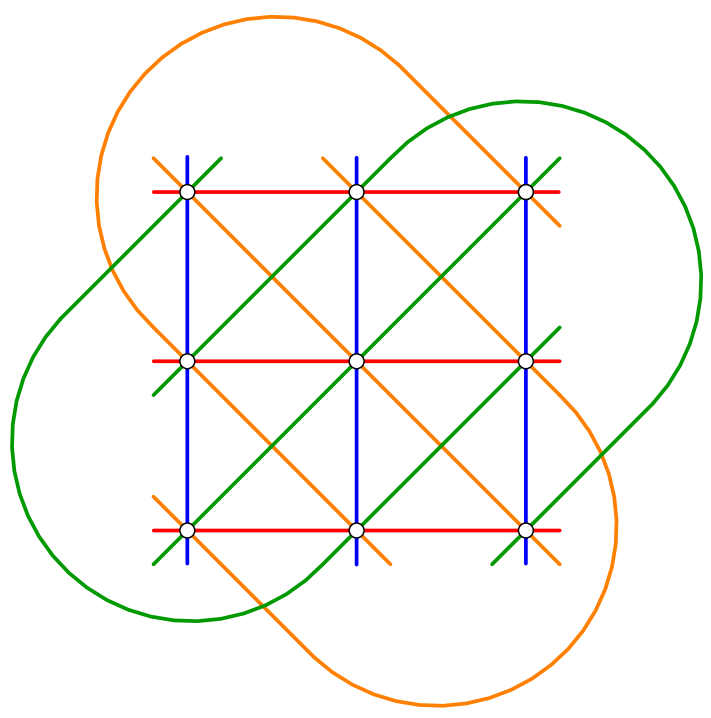

incident to $\lambda$ lines, while each of the $\ell$-lines is incident to $\pi$ points. As usual, we write $\left(n_{k}\right)$ for a configuration of the type $\left(n_{k}, n_{k}\right)$ (see Grünbaum 2009 as a main reference for configurations). Schroeter gave in Schroeter (1888) a proof that $\left(n_{3}\right)$ configurations can be realized in the real projective plane for $n \geq 9$. His construction relies on properties of point sets on cubic curves. Moreover, since all steps in his construction can be carried out with ruler alone, the corresponding configurations can also be geometrically realized in the rational plane.

As an application of Schroeter's construction we provide now a few configurations whose points belong to an elliptic curve.

To warm up, assume that $A, \bar{A}, B, \bar{B}, C, \bar{C}$ are six pairwise distinct points in the real projective plane such that the three pairs of points $A, \bar{A}, B, \bar{B}, C, \bar{C}$ are the pairs of opposite vertices of the same complete quadrilateral. In other words, assume that $A, B, C ; \bar{B}, \bar{A}, C ; A, \bar{B}, \bar{C}$; and $B, \bar{A}, \bar{C}$ are collinear. Then the six points $A, \bar{A}, B, \bar{B}$, $C, \bar{C}$ together with the four lines $A C, \bar{A} C, A \bar{C}, \bar{A} \bar{C}$ is a $\left(6_{2}, 4_{3}\right)$ configuration. We find this configuration on every elliptic curve $\Gamma_{6}$ which contains the torsion subgroup $\mathbb{Z} / 6 \mathbb{Z}$. The following table and Fig. 11 shows how we can assign the six points $A, \bar{A}$, $B, \bar{B}, C, \bar{C}$ to the elements of the group $\mathbb{Z} / 6 \mathbb{Z}$ :

\begin{tabular}{lllllll}
\hline $\mathbb{Z} / 6 \mathbb{Z}$ & 0 & 1 & 2 & 3 & 4 & 5 \\
$\Gamma_{6}$ & $\bar{C}=\mathscr{O}$ & $A$ & $B$ & $C=T$ & $\bar{A}$ & $\bar{B}$ \\
\hline
\end{tabular}

To construct a $\left(12_{4}, 16_{3}\right)$ configuration whose points are on an elliptic curve, we start with an elliptic curve $\Gamma_{2 \times 6}$ which contains the torsion subgroup $\mathbb{Z} / 2 \mathbb{Z} \times \mathbb{Z} / 6 \mathbb{Z}$, assign the six points $A, \bar{A}, B, \bar{B}, C, \bar{C}$ in a suitable way to the elements of the group 


\begin{tabular}{lcccccc}
$\mathbb{Z} / 6 \mathbb{Z}$ & 0 & 1 & 2 & 3 & 4 & 5 \\
\hline$\Gamma_{6}$ & $\bar{C}=\mathscr{O}$ & $A$ & $B$ & $C=T$ & $\bar{A}$ & $\bar{B}$
\end{tabular}

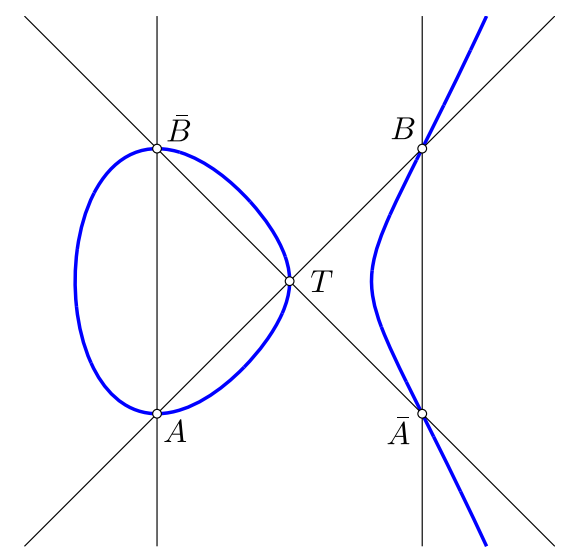

Fig. 11 A $\left(6_{2}, 4_{3}\right)$ configuration on an elliptic curve $\Gamma_{6}$

$\mathbb{Z} / 2 \mathbb{Z} \times \mathbb{Z} / 6 \mathbb{Z}$ (see the table below and Fig. 12), and construct with Schroeter's construction with these six points the points $D, \bar{D}, E, \bar{E}$, and $F, \bar{F}$ :

\begin{tabular}{lllllll}
\hline $\mathbb{Z} / 2 \mathbb{Z} \times \mathbb{Z} / 6 \mathbb{Z}$ & $(0,0)$ & $(0,1)$ & $(0,2)$ & $(0,3)$ & $(0,4)$ & $(0,5)$ \\
$\Gamma_{2 \times 6}$ & $\bar{D}=\mathscr{O}$ & $A$ & $B$ & $D=T$ & $\bar{A}$ & $\bar{B}$ \\
$\Gamma_{2 \times 6}$ & $\bar{F}$ & $C$ & $E$ & $F$ & $\bar{C}$ & $\bar{E}$ \\
$\mathbb{Z} / 2 \mathbb{Z} \times \mathbb{Z} / 6 \mathbb{Z}$ & $(1,0)$ & $(1,1)$ & $(1,2)$ & $(1,3)$ & $(1,4)$ & $(1,5)$ \\
\hline
\end{tabular}

Notice that $A, \bar{A}, B, \bar{B}, D, \bar{D}$, as well as $C, \bar{C}, E, \bar{E}, D, \bar{D}$, are the pairs of opposite vertices of a complete quadrilateral. These two complete quadrilaterals consist of 10 points and 8 lines. Together with the 2 points $F$ and $\bar{F}$, and the 4 lines $A E$, $\bar{A} \bar{E}, \bar{A} E, A \bar{E}$, we obtain a $\left(12_{4}, 16_{3}\right)$ configuration where all 12 points belong to an elliptic curve $\Gamma_{2 \times 6}$. $\left(12_{4}, 16_{3}\right)$ configurations have been discussed quite intensively in the literature (see, e.g., Gropp 1992; Mendelsohn et al. 1987; Metelka 1985 and the references therein), the more astonishing it is how easily Schroeter's construction yields this configuration on an elliptic curve.

As a further example of Schroeter's construction, we construct a $\left(24_{6}, 48_{3}\right)$ configuration whose points are on an elliptic curve. In order to simplify the notation, we introduce the following terminology: For 4 pairwise distinct points $P, \bar{P}, Q, \bar{Q}$, let $(P, Q: S)$ be an abbreviation for the construction of the two points $S, \bar{S}$, where

$$
S:=P Q \wedge \bar{P} \bar{Q} \text { and } \bar{S}:=P \bar{Q} \wedge \bar{P} Q
$$




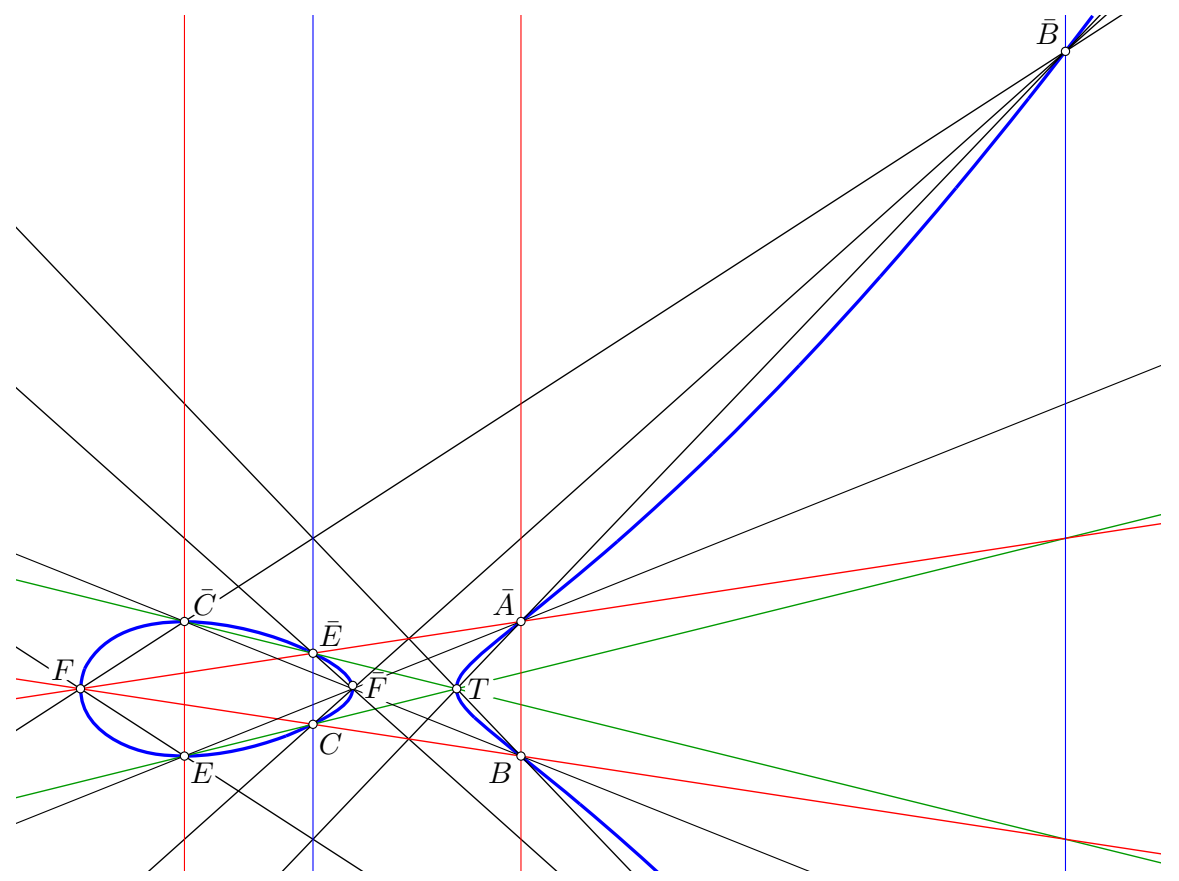

Fig. 12 A $\left(12_{4}, 16_{3}\right)$ configuration on an elliptic curve $\Gamma_{2 \times 6}$

Let $\Gamma_{26}$ be an elliptic curve which contains the torsion subgroup $\mathbb{Z} / 26 \mathbb{Z}$, and let us assign the points $\mathscr{O}, T, A, \bar{A}, B, \bar{B}, C, \bar{C}$ to the elements $0,13,2,15,6,19,8,21$, respectively. Now, we carry out the following constructions in the given order:
1. $(A, B: D)$
2. $(A, C: E)$
3. $(B, E: F)$
4. $(C, F: G)$
5. $(A, G: H)$
6. $(F, H: J)$
7. $(D, J: K)$
8. $(H, K: L)$
9. $(C, L: M)$
10. $(B, K: M)$
11. $(E, J: M)$
12. $(D, G: L)$

All together, we have constructed 24 points (including the six points $A, \bar{A}, B, \bar{B}$, $C, \bar{C})$ :

\begin{tabular}{lllllllllllll}
\hline $\mathbb{Z} / 26 \mathbb{Z}$ & 2 & 4 & 6 & 8 & 10 & 12 & 14 & 16 & 18 & 20 & 22 & 24 \\
\hline$\Gamma_{26}$ & $A$ & $F$ & $B$ & $C$ & $H$ & $J$ & $G$ & $E$ & $D$ & $L$ & $K$ & $M$ \\
$\Gamma_{26}$ & $\bar{A}$ & $\bar{F}$ & $\bar{B}$ & $\bar{C}$ & $\bar{H}$ & $\bar{J}$ & $\bar{G}$ & $\bar{E}$ & $\bar{D}$ & $\bar{L}$ & $\bar{K}$ & $\bar{M}$ \\
$\mathbb{Z} / 26 \mathbb{Z}$ & 15 & 17 & 19 & 21 & 23 & 25 & 1 & 3 & 5 & 7 & 9 & 11 \\
\hline
\end{tabular}

In addition, we have constructed 48 lines and each of the 24 points is incident to 6 lines, while each of the 48 -lines is incident to 3 points. Therefore, we have a $\left(24_{6}, 48_{3}\right)$ configuration all whose points are on the elliptic curve $\Gamma_{26}$. Notice that if we just consider the 12 points $A, B, C, \ldots, M$ and the 12 lines $A B D, A C E, B E F, \ldots, D G L$, 
then we obtain a $\left(12_{3}\right)$ configuration (i.e., a $\left(12_{3}, 12_{3}\right)$ configuration), all whose points are on the elliptic curve $\Gamma_{26}$.

Acknowledgements We would like to thank the referee for his or her careful reading and the suggestions that helped improve the substance of the article.

Funding Open Access funding provided by ETH Zurich.

Open Access This article is licensed under a Creative Commons Attribution 4.0 International License, which permits use, sharing, adaptation, distribution and reproduction in any medium or format, as long as you give appropriate credit to the original author(s) and the source, provide a link to the Creative Commons licence, and indicate if changes were made. The images or other third party material in this article are included in the article's Creative Commons licence, unless indicated otherwise in a credit line to the material. If material is not included in the article's Creative Commons licence and your intended use is not permitted by statutory regulation or exceeds the permitted use, you will need to obtain permission directly from the copyright holder. To view a copy of this licence, visit http://creativecommons.org/licenses/by/4.0/.

\section{References}

Chasles, M.: Aperçu historique sur l'origine et le développement des méthodes en géométrie. Éditions Jacques Gabay, Sceaux. Reprint of the 1837 original (1989)

Coxeter, H.S.M., Greitzer, S.L.: Geometry revisited. New Mathematical Library, vol. 19. Random House Inc, New York (1967)

Gropp, H.: The construction of all configurations $\left(12_{4}, 16_{3}\right)$. In: Fourth Czechoslovakian Symposium on Combinatorics, Graphs and Complexity (Prachatice, 1990), volume 51 of Ann. Discrete Math., pp. 85-91. North-Holland, Amsterdam (1992)

Grünbaum, B.: Configurations of Points and Lines. Graduate Studies in Mathematics, vol. 103. American Mathematical Society, Providence, RI (2009)

Halbeisen, L., Hungerbühler, N.: Pairing pythagorean pairs. J. Number Theory (2021). arxiv.org/abs/2101.08163

Halbeisen, L., Hungerbühler, N., Zargar, A.S.: New para-metrisations of elliptic curves with torsion groups $\mathbb{Z} / 10 \mathbb{Z}, \mathbb{Z} / 12 \mathbb{Z}$, and $\mathbb{Z} / 14 \mathbb{Z}$. (2021) arXiv:2106.06861

Kubert, D.S.: Universal bounds on the torsion of elliptic curves. Proc. Lond. Math. Soc. (3) 33(2), 193-237 (1976)

Mendelsohn, N.S., Padmanabhan, R., Wolk, B.: Designs embeddable in a plane cubic curve. (Part 2 of Planar projective configurations). Note Mat. 7(1), 113-148 (1987)

Mendelsohn, N.S., Padmanabhan, R., Wolk, B.: Straight edge constructions on planar cubic curves. C. R. Math. Rep. Acad. Sci. Canada 10(2), 77-82 (1988)

Metelka, V.: On two special configurations $\left(12_{4}, 16_{3}\right)$. Časopis Pěst. Mat. 110(4), 351-355 (1985)

Rabarison, F.P.: Structure de torsion des courbes elliptiques sur les corps quadratiques. Acta Arith. 144(1), 17-52 (2010)

Schroeter, H.: Die Theorie der ebenen Curven dritter Ordnung. B. G. Teubner, Leipzig (1888)

Schröter, H.: Ueber eine besondere Curve $3^{\text {ter }}$ Ordnung und eine einfache Erzeugungsart der allgemeinen Curve $3^{\text {ter }}$ Ordnung. Math. Ann. 5(1), 50-82 (1872)

Schröter, H.: Ueber Curven dritter Ordnung. Math. Ann. 6(1), 85-111 (1873)

Publisher's Note Springer Nature remains neutral with regard to jurisdictional claims in published maps and institutional affiliations. 